\title{
Medical Services at an International Summer Camp Event Under Hot and Humid Conditions: Experiences From the 23rd World Scout Jamboree, Japan
}

\author{
Takemasa Watanabe, MD, PhD; Keiji Mizutani, MD, PhD; Toshiyasu Iwai, MD; Hiroshi Nakashima, BPharm
}

From the Medical Team of the Scout Association of Japan, Tokyo, Japan (Drs Watanabe, Mizutani, Iwai, and Nakashima); the Graduate School of Health and Sport Science, Chukyo University, Toyota, Japan (Dr Watanabe); the Department of Gastroenterological Medicine, Ichinomiya Municipal Hospital, Ichinomiya, Japan (Dr Mizutani); the Medical Care Center in Remote Rural Areas, Kagawa Prefectural Central Hospital, Takamatsu, Japan (Dr Iwai); and the Medical Safety Management Office, Toukai Central Hospital, Kagamihara, Japan (Dr Nakashima).

Introduction-The 23rd World Scout Jamboree (WSJ) was a 10-day summer camp held in Japan in 2015 under hot and humid conditions. The attendees comprised 33,628 people from 155 countries and territories. The aim of this study was to examine the provision of medical services under such conditions and to identify preventive factors for major diseases among long-term campers.

Methods-Data were obtained from WSJ medical center records and examined to clarify the effects of age, sex, and period on visit frequencies and rates.

Results-Medical records from 3215 patients were examined. Daytime temperatures were $31.5 \pm 3.2^{\circ} \mathrm{C}$ and relative humidity was $61 \pm 13 \%$ (mean $\pm \mathrm{SD}$ ). The initial visit rates among scouts and adults were 72.2 and 77.2 per 1000 persons, respectively. No significant age difference was observed in the initial visit rate; however, it was significantly higher among female patients than male patients. Significant differences were also seen in the adjusted odds ratios by age, sex, and period for disease distributions of initial visit frequencies. In addition, a higher initial visit frequency for heat strain-related diseases was seen among the scouts. Initial visit frequencies for heatstroke and/or dehydration increased just after opening day and persisted until closing day.

Conclusions-Our findings suggest the importance of taking effective countermeasures against heat strain, fatigue, and unsanitary conditions at the WSJ. Medical services staff should take attendees' age, sex, and period into consideration to prevent heat strain-related diseases during such camps under hot and humid conditions.

Keywords: hot and humid environment, preventive factor

\section{Introduction}

The 23rd World Scout Jamboree (WSJ) was held at Kirara-hama, Yamaguchi Prefecture, Japan, in the summer of 2015. The attendees comprised 33,628 people from 155 countries and territories. ${ }^{1}$ The WSJ aims to be attended once in a lifetime by scouts, regardless of sex, who belong to the World Organization of the Scout Movement. Whether exploring challenges in international development that affect all nations and societies at the Global Development Village or simply sharing a meal

Corresponding author: Takemasa Watanabe, MD, PhD, Executive Secretary, Medical Team of the Scout Association of Japan, Tokyo 113-0033, Japan; e-mail: t_nabe@sass.chukyo-u.ac.jp.

Submitted for publication May 2017.

Accepted for publication January 2018. with newfound friends from far-off parts of the world, the WSJ is a unique environment in which scouts are encouraged to develop as global citizens who can inspire positive change in their communities. ${ }^{2}$

Medical care plays a crucial role in the WSJ by helping to ensure that participants can carry out camping and outdoor activities safely and comfortably. Compared with the 22nd WSJ, which was held in Sweden in 2011, the 23rd WSJ was held under extremely hot and humid conditions in Japan. Furthermore, most attendees had to travel much further before arriving at the WSJ site. Several reports ${ }^{4-6}$ on long-term camping have highlighted the importance of first aid in preventing and treating trauma and infectious diseases; however, only a few studies regarding the organization and implementation of camp-wide health care have been conducted. ${ }^{3}$ 
Based on our experience at the 16th Nippon Jamboree, which was held in 2013 at the same site during the same season (summer) as a precursor to the 23rd WSJ, the primary focus of medical care was the prevention of heat stroke and skin diseases, including insect bites.

The objectives of the present study were to summarize experiences in relation to the provision of medical services for the 23rd WSJ and to identify preventive factors for major diseases among long-term campers under hot and humid conditions.

\section{Methods}

\section{MEDICAL CARE AT THE 23RD WSJ}

Medical facilities, namely the WSJ medical center and first aid points, were set up to provide care in the event of illness or injury. The WSJ medical center provided the most comprehensive level of care available at the WSJ site. If advanced diagnoses or complicated surgical procedures were required, patients were transferred to a local off-site hospital. First aid points were established at 3 hub campsites to provide treatment for minor illnesses and injuries. The WSJ fee did not cover any clinical examinations and/or treatments at off-site medical facilities; in such cases, contingent unit leaders needed to accompany the patient and arrange their own transportation. A dedicated ambulance was specifically prepared and kept aside for urgent cases. English, French, and Japanese interpreters were also available at the WSJ medical center. The WSJ medical center was available from July 25 to August 9, 2015; the WSJ programs and events started on July 29 and ended on August 7, 2015.

The WSJ medical center, which was set up in a large tent (floor area: $10 \mathrm{~m} \times 35 \mathrm{~m}$ ) with air conditioners, included the following: a reception area; a waiting room; 4 examination rooms; 2 treatment rooms; 4 observation rooms, each with a capacity for 10 patients; a blood laboratory; and a pharmacist's office. The daily staff consisted of at least 10 medical doctors, 2 pharmacists, 15 nurses, and 20 clerks. Six-member groups of doctors and nurses were assigned to each first aid point. In total, 632 medical workers staffed the WSJ medical center.

\section{ENVIRONMENTAL CONDITIONS OF CAMP LIFE}

The camp area was located near the coast on grass or dirt surfaces. The campsite was approximately $1 \mathrm{~km} \times 3 \mathrm{~km}$ and covered an area of about 286 ha. In the 8 years before the WSJ, the weather around the WSJ site from July 31 to August 9 tended to be sunny and clear with only occasional clouds and rain showers. During the 23rd WSJ events (the WSJ medical center was operational from July 25 to August 9), the weather was sunny and clear with no rain. High temperatures persisted throughout the entire period, with mean daytime and nighttime temperatures of $31.5 \pm 3.2^{\circ} \mathrm{C}$ and $26.7 \pm 2.0^{\circ} \mathrm{C}$, respectively, as recorded in nearby Yamaguchi city. The mean daytime and nighttime relative humidity was 61 $\pm 13 \%$ and $79 \pm 11 \%$, respectively. Compared with the 22nd WSJ in Sweden in $2011,{ }^{3}$ both mean daytime and nighttime temperatures throughout the 10-day camp were about $10^{\circ} \mathrm{C}$ higher. Daytime changes in mean wet-bulb globe temperature (WBGT) values from July 31 to August 8 were measured in front of the WSJ medical center. WBGT values were determined at $1.5 \mathrm{~m}$ in height off the dirt ground using an automatic measurement device (WBGT 213B; Midori Anzen Co, Tokyo, Japan). The average maximum WBGT value was $31.4 \pm 1.1^{\circ} \mathrm{C}$ (maximum: $33.3^{\circ} \mathrm{C}$, minimum: $30.0^{\circ} \mathrm{C}$ ).

\section{ANALYSIS PROCEDURES}

i) An epidemiologist licensed as a medical doctor checked the accuracy of the diagnoses from all medical records before data analysis.

ii) Initial and return visit frequencies during the period in which the WSJ medical center was operational were calculated. The visit rate was calculated using the following formula: visit rate (per 1000 persons)= number of visits/number of participants x 1000 persons. The daily initial visit rate was calculated using the following formula: daily initial visit rate (per 1000 persons per day) $=$ number of daily initial visits/number of participants/1 day x 1000 persons.

iii) Age, sex, and period were applied as explanatory variables. Age was categorized as "scouts" (14-17 years) and "adults" (18 years and older). Sex was categorized as "female" and "male." Period was categorized as "first half" (a consultation date from July 25 to August 2) and "second half" (a consultation date from August 3 to August 9).

iv) Multivariate logistic regression analysis was applied to assess the independent effects of age, sex, and period on initial visit frequencies among the 2466 initial-visit patients with no missing values. Diseases with significant odds ratios (ORs) were extracted.

v) Initial visit frequencies for heat stroke and/or dehydration were calculated and illustrated by age, sex, and consultation date.

vi) PASW Statistics for Windows (Version 23.0; IBM, Armonk, NY) was used for statistical analysis. Significant differences between groups were detected using the $\chi^{2}$ test. Statistical significance was set at $P<0.05$. 


\section{ETHICAL APPROVAL AND CONSENT}

This study was approved by the Human Subjects Committee at Chukyo University Graduate School of Health and Sport Sciences (Protocol Number: 2016-2). Informed consent was not required for this type of study because it used anonymous data.

\section{Results}

A total of 3215 patients (2142 scouts, 1073 adults) required medical assistance at the WSJ medical center during the period in which it was operational. A total of 122 patients who required clinical examination and/or treatment (eg, suspicion of bone fracture, treatment requiring hospitalization) were transferred to an off-site hospital near the WSJ.

The total visit rates were 95.6 per 1000 persons (female: 117.1, male: 77.3) among scouts and 100.0 per 1000 persons (female: 125.6, male: 86.5) among adults. The initial visit rates were 72.2 per 1000 persons (female: 93.1, male: 58.3) among scouts and 77.2 per 1000 persons (female: 101.2, male: 86.5) among adults. No significant differences were observed between scouts and adults in total or initial visit rates; however, initial visit rates were significantly higher for female patients compared with male patients. The visit frequencies of the 23rd WSJ attendees during the period in which the WSJ medical center was operational are shown in Figure 1. A decrease in all-visit frequency was observed on August 1 ; this decrease corresponded with the cessation of all activities outside the WSJ area that involved bussing (offsite programs), such as mountain and water activities and tours, by the WSJ risk management center to allow the attendees to rest and to prevent heat stroke.

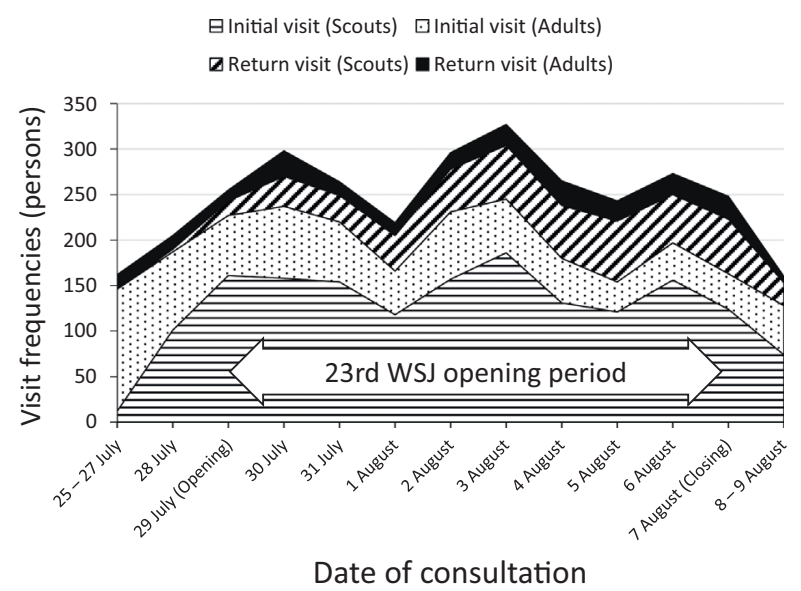

Figure 1. Changes in visit frequencies during the period in which the 23rd World Scout Jamboree medical center was operational.
The mean daily initial visit rates among scouts and adults during the entire open period of the 23rd WSJ, from July 29 to August 7, were $6.4 \pm 0.9$ and $5.2 \pm 1.3$ per 1000 persons per day, respectively. Before the opening day of the WSJ medical center, the daily initial visit rates were higher among adults than among scouts. In the first half of the WSJ, the daily initial visit rates among scouts were similar to those among adults; however, in the second half, the daily initial visit rates among scouts were higher. No differences in daily initial visit rates were observed between the sexes in either scouts or adults during the period in which the WSJ medical center was operational.

Disease distributions of initial visit frequencies during the entire period in which the WSJ medical center was operational are shown in Table 1. In scouts, diseases with high initial visit frequency were i) heat stroke and/or dehydration $(n=262)$; ii) common cold, pharyngitis, and/ or upper respiratory inflammation $(n=175)$; iii) incised wound and/or laceration ( $\mathrm{n}=124)$; iv) diarrhea and/or enteritis $(n=107)$; v) cellulitis and/or paronychia $(n=97)$; and vi) insect bite and/or sting $(n=87)$. Those in adults were i) common cold, pharyngitis, and/or upper respiratory inflammation $(n=101)$; ii) heat stroke and/or dehydration $(\mathrm{n}=86)$; iii) blister and/or shoe sore $(\mathrm{n}=78)$; iv) contact dermatitis and/or eczema ( $n=57)$; v) insect bite and/or sting $(n=50)$; and vi) diarrhea and/or enteritis $(n=45)$.

Adjusted ORs and 95\% confidence intervals (CIs) of risk factors, namely age (scouts/adults), sex (female/ male), and period (first half/second half), for major diseases based on the multivariate logistic regression analysis are shown in Table 2. The following diseases were significantly found to be more likely to occur in scouts versus adults: heat stroke and/or dehydration, fatigue and/or headaches, incised wound and/or laceration, and impetigo (OR $>1)$; and less likely to occur: blister and/or shoe sore $(\mathrm{OR}<1)$. The following diseases were significantly found to be more likely to occur in females versus males: sprain, fracture and/or dislocation, and panic attack and/or hyperventilation $(\mathrm{OR}>1)$; and less likely to occur: incised wound and/ or laceration, bruise and/or contusion, cellulitis and/or paronychia $(\mathrm{OR}<1)$. The following diseases were significantly found to be more likely to occur during the first half of the event versus second half: heat stroke and/or dehydration, incised wound and/or laceration, and contact dermatitis and/or eczema $(\mathrm{OR}>1)$; and less likely to occur: common cold, pharyngitis and/or upper respiratory inflammation, stomatitis and/or cheilitis, and impetigo $(\mathrm{OR}<1)$.

The following special cases were also experienced: 2 cases of deep vein thrombosis in the early period of the WSJ; 3 cases of hand, foot, and mouth disease; 2 cases 
Table 1. Disease distributions of initial visit frequencies during the period in which the WSJ medical center was operational

\begin{tabular}{|c|c|c|c|}
\hline \multirow[b]{2}{*}{ Case type } & \multirow[b]{2}{*}{ Major diseases } & \multicolumn{2}{|c|}{ Initial visit frequency } \\
\hline & & Scouts (female/male) & Adults (female/male) \\
\hline \multirow[t]{9}{*}{ Internal medical cases } & $\begin{array}{l}\text { Common cold, pharyngitis, and/or } \\
\text { upper respiratory inflammation }\end{array}$ & $175(76 / 99)$ & $101(40 / 61)$ \\
\hline & Asthma & $17(7 / 10)$ & $6(3 / 3)$ \\
\hline & Stomachache and/or gastritis & $68(37 / 31)$ & $24(11 / 13)$ \\
\hline & Diarrhea and/or enteritis & $107(45 / 62)$ & $45(22 / 23)$ \\
\hline & Constipation & $25(15 / 10)$ & $11(4 / 7)$ \\
\hline & Heat stroke and/or dehydration & $262(135 / 125)$ & $86(42 / 44)$ \\
\hline & Fatigue and/or headache & $69(37 / 32)$ & $19(9 / 10)$ \\
\hline & Stomatitis and/or cheilitis & $46(23 / 22)$ & $12(2 / 10)$ \\
\hline & Other $^{a}$ & $87(49 / 37)$ & $62(33 / 29)$ \\
\hline \multirow[t]{4}{*}{ Traumatic cases } & Incised wound and/or laceration & $124(42 / 79)$ & $41(13 / 28)$ \\
\hline & Bruise and/or contusion & $50(14 / 35)$ & $15(7 / 8)$ \\
\hline & Sprain, fracture, and/or dislocation ${ }^{b}$ & $78(46 / 30)$ & $25(14 / 11)$ \\
\hline & Other & $49(25 / 24)$ & $27(12 / 15)$ \\
\hline \multirow[t]{9}{*}{ Dermatological cases } & Contact dermatitis and/or eczema & $85(44 / 40)$ & $57(27 / 30)$ \\
\hline & Insect bite and/or sting ${ }^{c}$ & $87(39 / 45)$ & $50(29 / 21)$ \\
\hline & Sunburn and/or solar dermatitis & $81(40 / 41)$ & $56(29 / 27)$ \\
\hline & Blister and/or shoe sore & $65(39 / 26)$ & $78(30 / 48)$ \\
\hline & Scald and/or burn & $23(11 / 12)$ & $6(2 / 4)$ \\
\hline & Cellulitis and/or paronychia & $97(37 / 59)$ & $62(20 / 24)$ \\
\hline & Impetigo & $33(16 / 17)$ & $3(1 / 2)$ \\
\hline & Heat rash & $24(9 / 15)$ & $17(6 / 11)$ \\
\hline & Other $^{d}$ & $53(24 / 28)$ & $31(11 / 20)$ \\
\hline \multirow[t]{3}{*}{ Otorhinological cases } & Otitis media & $26(10 / 16)$ & $5(3 / 2)$ \\
\hline & Nasal bleeding & $10(4 / 6)$ & $10(4 / 6)$ \\
\hline & Other $^{e}$ & $22(10 / 12)$ & $14(4 / 10)$ \\
\hline \multirow[t]{2}{*}{ Ophthalmological cases } & Conjunctivitis and/or corneitis & $35(18 / 16)$ & $25(8 / 17)$ \\
\hline & Other & $45(20 / 15)$ & $11(9 / 2)$ \\
\hline Dental cases & Total cases & $45(23 / 22)$ & $15(3 / 12)$ \\
\hline \multirow[t]{2}{*}{ Psychiatry and psychological cases } & Panic attack and/or hyperventilation & $25(18 / 7)$ & $5(4 / 1)$ \\
\hline & Other & $19(13 / 6)$ & $4(4 / 0)$ \\
\hline
\end{tabular}

WSJ, World Scout Jamboree.

${ }^{a}$ Other internal medical cases included 3 cases of hand, foot, and mouth disease; 2 cases of influenza B; 2 cases of deep vein thrombosis; and 4 cases of epilepsy.

${ }^{b}$ Sprain, fracture, and/or dislocation cases included 24 suspected cases of fracture and 8 cases of joint dislocation or subluxation.

${ }^{c}$ Insect bite and/or sting cases included 19 cases of Paederus dermatitis.

${ }^{d}$ Other dermatological cases included 19 cases of jellyfish stings, 12 cases of athlete's foot, 7 cases of urticaria, and 6 cases of herpes infection.

${ }^{e}$ Other otorhinological cases included 10 cases of impacted ear wax.

of influenza B; and 6 cases of herpes infection in the middle period of the WSJ.

Initial visit frequencies of heat stroke and/or dehydration were 135 in female scouts, 125 in male scouts, 42 in female adults, and 44 in male adults. Significant ORs for heat stroke and/or dehydration on initial visit were 1.79 by age and 1.85 by period. The initial visit frequencies for heat stroke and/or dehydration are shown in Figure 2. These values increased on July 29 and 30, just after the opening day of the 23rd WSJ, decreased rapidly on August 1, increased again after August 1, and then persisted until closing day.

\section{Discussion}

\section{CHANGES IN VISIT FREQUENCIES AND RATES TO THE 23RD WSJ MEDICAL CENTER}

The total visit rate to the 23rd WSJ medical center among scouts was 95.6 per 1000 persons. Considering that the estimated frequency of first aid point visits was about 2000 persons, the total visit rate for all WSJ medical services was much higher than the rate of 80.6 per 1000 persons (3229 from among 40,061 attendees) seen during the 22nd WSJ in Sweden in 2011, which was held in the same season for a similar period and at a 
Table 2. Adjusted ORs and 95\% CIs for major diseases by age, sex, and period among initial visitors during the period in which the WSJ medical center was operational

\begin{tabular}{|c|c|c|c|}
\hline \multirow[b]{2}{*}{ Major diseases } & \multicolumn{3}{|c|}{ Explanatory variables } \\
\hline & $\begin{array}{l}\text { Age }(\text { scout/adult }) \\
\text { OR }(95 \% \text { CI })\end{array}$ & $\begin{array}{l}\text { Sex }(\text { female/male }) \\
\quad \text { OR }(95 \% \text { CI })\end{array}$ & $\begin{array}{l}\text { Period (first half/second half) } \\
\text { OR }(95 \% \text { CI) }\end{array}$ \\
\hline $\begin{array}{l}\text { Common cold, pharyngitis, and/or upper } \\
\text { respiratory inflammation }\end{array}$ & NS & NS & $0.47(0.36-0.61)$ \\
\hline Asthma & NS & NS & NS \\
\hline Stomachache and/or gastritis & NS & NS & NS \\
\hline Diarrhea and/or enteritis & NS & NS & NS \\
\hline Constipation & NS & NS & NS \\
\hline Heat stroke and/or dehydration & $1.79(1.38-2.33)$ & NS & $1.85(1.45-2.37)$ \\
\hline Fatigue and/or headache & $1.87(1.12-3.11)$ & NS & NS \\
\hline Stomatitis and/or cheilitis & NS & NS & $0.58(0.34-0.99)$ \\
\hline Incised wound and/or laceration & $1.80(1.24-2.61)$ & $0.51(0.36-0.71)$ & $1.99(1.40-2.82)$ \\
\hline Bruise and/or contusion & NS & $0.53(0.31-0.89)$ & NS \\
\hline Sprain, fracture, and/or dislocation & NS & $1.58(1.05-2.36)$ & NS \\
\hline Contact dermatitis and/or eczema & NS & NS & $1.50(1.05-2.14)$ \\
\hline Insect bite and/or sting & NS & NS & NS \\
\hline Sunburn and/or solar dermatitis & NS & NS & NS \\
\hline Blister and/or shoe sore & $0.38(0.27-0.53)$ & NS & NS \\
\hline Scald and/or burn & NS & NS & NS \\
\hline Cellulitis and/or paronychia & NS & $0.59(0.42-0.82)$ & NS \\
\hline Impetigo & $4.66(1.42-15.31)$ & NS & $0.24(0.11-0.54)$ \\
\hline Heat rash & NS & NS & NS \\
\hline Otitis media & NS & NS & NS \\
\hline Conjunctivitis and/or corneitis & NS & NS & NS \\
\hline Dental cases & NS & NS & NS \\
\hline Panic attack and/or hyperventilation & NS & $2.91(1.29-6.57)$ & NS \\
\hline
\end{tabular}

OR, odds ratio; NS, not significant.

similar scale compared with the 23rd WSJ. During the WSJ open period from July 29 to August 7, the daily visit frequency was around 300 persons. A decline observed on August 1 was thought to have been the

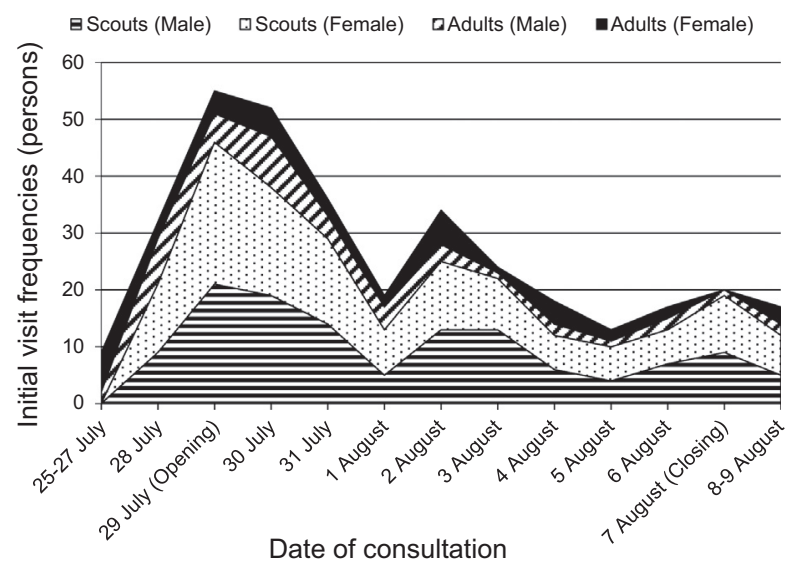

Figure 2. Changes in initial visit frequencies for heatstroke and/or dehydration during the period in which the 23rd World Scout Jamboree medical center was operational. result of a cessation of all off-site programs conducted far from the WSJ site by order of the WSJ risk management center to allow the attendees to rest near the campsite and to prevent heat stroke. Clear and sunny weather with no precipitation continued throughout the remainder of the 23rd WSJ, with daytime temperatures mostly staying above $30^{\circ} \mathrm{C}$, which is equivalent to a WBGT value of $28^{\circ} \mathrm{C}$. Under these conditions, the restriction of sports and outdoor activities is recommended. ${ }^{7-9}$

The decline in the daily initial visit rate on August 1 was seen across all age and sex groups. Total initial visits were buffered by the cessation of all activities outside the WSJ area, and the primary threats were heat and humidity as opposed to storms. A higher initial visit rate in adults before the WSJ opening period may have been the result of differences between adults and scouts regarding arrival dates and workloads. In the first half of the period, initial visit rates among age and sex groups were nearly identical; however, in the second half, initial visit rates in both female and male scouts were higher than those in adults and continued to be higher until 
closing day. This suggests that the conditions for both female and male scouts attending the WSJ did not improve over the 10-day period.

\section{DISEASE DISTRIBUTION OF INITIAL VISIT FREQUENCIES DURING THE PERIOD IN WHICH THE 23RD WSJ MEDICAL CENTER WAS OPERATIONAL}

Diseases associated with higher initial visit frequency in scouts were heat stroke and/or dehydration; common cold, pharyngitis, and/or upper respiratory inflammation; incised wound and/or laceration; and diarrhea and/or enteritis. Diseases in adults were common cold, pharyngitis, and/or upper respiratory inflammation; heat stroke and/or dehydration; blister and/or shoe sore; contact dermatitis and/or eczema; insect bite and/or sting; and diarrhea and/or enteritis. Hot and humid environments increase the risk of initial visit from heat stroke and/or dehydration, ${ }^{10,11}$ and the combined effects of heat strain, dehydration, and exercise under excessively hot and humid environments can lead to fatigue and impaired immunity, ${ }^{12-14}$ subsequently resulting in an increased risk of infectious disease, skin disease, and other related injuries.

Based on the results of multivariate logistic regression analysis involving 2466 initial visitors, diseases dominant in scouts were impetigo, fatigue and/or headaches, incised wound and/or laceration, and heat stroke and/or dehydration. This suggests that scouts were adversely affected by the severe weather conditions and the uncomfortable and unsanitary conditions of camp life. On the other hand, blister and/or shoe sore was dominant in adults, suggesting that most of the adult attendees walked long distances and had lengthy exposure to the sun. Diseases dominant in females were sprain, fracture and/or dislocation, and panic attack and/or hyperventilation, while those in males were common cold, pharyngitis, and/or upper respiratory inflammation; bruise and/or contusion; and incised wound and/or laceration. The differences observed between females and males in our study are similar to those reported in studies on "sports injuries" 15,16 and international military deployments. ${ }^{17}$ These findings suggest potential sex differences in musculoskeletal, biomechanical, and physiological characteristics. Diseases dominant in the first half of the WSJ were heat stroke and/or dehydration, incised wound and/or laceration, and contact dermatitis and/or eczema, whereas those dominant in the second half were common cold; pharyngitis, and/or upper respiratory inflammation; stomatitis and/or cheilitis; and impetigo. The diseases dominant in the first half of the WSJ may be explained by the suggestion that successful acclimation to heat stress increases workload capacity in a hot and humid environment, reduces rectal temperature during exercise, and diminishes the risk for exertional heat-related illnesses. ${ }^{18,19}$ The diseases dominant in the second half of the WSJ may be explained in terms of immunological hypofunction through fatigue and unsanitary conditions under hot and humid conditions.

Regarding special cases requiring an emergency response, we observed a case of deep vein thrombosis just after the WSJ medical center opening day. This suggested that deep vein thrombosis might have been brought out by the long travel duration. The transmission of an infectious disease occurred in the middle period of the WSJ through contact with strangers during an off-site program in which scouts travelled far from the WSJ site by bus.

\section{CHANGES IN INITIAL VISIT FREQUENCIES FOR HEAT STROKE AND/OR DEHYDRATION}

Heat stroke and dehydration has been documented among athletes and wilderness enthusiasts who engage in long periods of strenuous physical activities in hot environments. ${ }^{9,20,21}$ In our study, initial visit frequency for heat stroke and/or dehydration were higher in scouts than in adults, and no significant sex differences were observed. The higher metabolic cost of locomotion in children provides an added strain on the thermoregulatory system during exercise in the heat, ${ }^{22}$ and the magnitude of adaptation is determined by the intensity, duration, frequency, and number of heat exposures. ${ }^{10}$ The age differences observed in the initial visits for heat stroke may have resulted from deficient exposure to heat stress and exercise just before the 23rd WSJ. Poor preparatory conditions among scouts could have led to the increase seen in initial visits for heat stroke and dehydration just after opening day, which persisted until closing day owing to the severely hot and humid environment.

\section{LIMITATIONS}

First, because our findings regarding preventive factors for major diseases were based on a single international camp event, more case studies on similar events should be conducted in the future. Second, only 1 medical doctor was in charge of checking the accuracy of diagnoses for all 3215 medical records. At least in principle, these types of records need to be thoroughly double-checked by multiple personnel; however, for this study, only 1 physician was available. Third, visits to first aid points were not included in this analysis; therefore, the number of heat-related morbidities might have been underestimated. Fourth, total camper days could not be used as the denominator for visit rates because the exact arrival and departure dates of participants were not known. Fifth, the environmental 
exposure of each attendee could not be assessed because the participation rates in various kinds of activity programs were unknown. Finally, the emergency medical area was set up to handle a population of about 300,000 in Japan, and the sudden temporary influx of more than 30,000 people imposed a large workload on off-site medical facilities around the WSJ site. Therefore, it is difficult to discuss adequate preparation for medical centers for longterm camp events similar to the 23rd WSJ.

\section{Conclusions}

In the present study, the medical records of 3215 attendees of the 23rd WSJ who required medical care were analyzed to identify preventive factors for major diseases in long-term camping life under hot and humid conditions. The results suggest the importance of effective countermeasures against heat strain, fatigue, and unsanitary conditions at an international summer camp. In addition to the camp period, medical services staff should take age, sex, and period into consideration to prevent heat-related diseases during such camps in hot and humid conditions.

Acknowledgments: The authors thank all members of the international service team who staffed the medical section as well as the entire staff of the 23rd World Scout Jamboree.

Author Contributions: Study concept and design (TW, KM); acquisition of the data (TW, KM, TI, HN); analysis of the data (TW); drafting of the manuscript (TW, KM); and approval of the final manuscript (TW, KM).

Financial/Material Support Statement: None.

Disclosure Statements: None.

\section{References}

1. Scout Association of Japan website. About the 23rd World Scout Jamboree, 2014. Available at: http://www.23wsj.jp/ about-23wsj.html. Accessed November 25, 2017.

2. Scout Association of Japan website. 23rd World Scout Jamboree Bulletin \#04, 2014. Available at: http://www. 23wsj.jp/assets/23wsj_bulletin_04_e.pdf. Accessed November $25,2017$.

3. Jammer I, Andersson CA, Olinder AL, Selander B, Wallinder AE, Hansson SR. Medical services of a multicultural summer camp event: experiences from the 22nd World Scout Jamboree, Sweden 2011. BMC Health Serv Res. 2013;13:187-92.

4. Yard EE, Scanlin MM, Erceg LE, Powell GM, Wilkins JR 3rd, et al. Illness and injury among children attending summer camp in the United States, 2005. Pediatrics. 2006;118(5):1342-9.

5. Key JD. Illness and injuries at summer camp. South Med J. 1997;90(5):489-92.

6. Goldlust E, Walton E, Stanley R, Yard E, Garst B, Comstock RD, et al. Injury patterns at US and Canadian overnight summer camp: first year of the Healthy Camp study. Inj Prev. 2009;15(6):413-7.

7. Kawahara T. Exercise guideline for prevention of heat injury (Japanese Sports Association) [in Japanese]. Nihon Rinsho. 2012;70(6):1029-32.

8. Binkley HM, Beckett J, Casa DJ, Kleiner DM, Plummer PE. National Athletic Trainers' Association position statement: exertional heat illnesses. J Athl Train. 2002;37(3):329-43.

9. Armstrong LE, Casa DJ, Millard-Stafford M, Moran DS, Pyne SW, Roberts WO. American College of Sports Medicine position stand. Exertional heat illness during training and competition. Med Sci Sports Exerc. 2007;39(3):556-72.

10. Périard JD, Racinais S, Sawka MN. Adaptations and mechanisms of human heat acclimation: applications for competitive athletes and sports. Scand J Med Sci Sports. 2015;25(Suppl 1):20-38.

11. Hancock PA, Ross JM, Szalma JL. A meta-analysis of performance response under thermal stressors. Hum Factors. 2007;49(5):851-77.

12. Mitchell JB, Dugas JP, McFarlin BK, Nelson MJ. Effect of exercise, heat stress, and hydration on immune cell number and function. Med Sci Sports Exerc. 2002;34(12): 1941-50.

13. Brenner I, Shek PN, Zamecnik J, Shephard RJ. Stress hormones and the immunological responses to heat and exercise. Int J Sports Med. 1998;19(2):130-43.

14. Pilcher JJ, Nadler E, Busch C. Effects of hot and cold temperature exposure on performance: a meta-analytic review. Ergonomics. 2002;45(10):682-98.

15. Holden S, Boreham C, Delahunt E. Sex differences in landing biomechanics and postural stability during adolescence: a systematic review with meta-analyses. Sports Med. 2016;46(2):241-53.

16. Schwebel DC, Brezausek CM. Child development and pediatric sport and recreational injuries by age. J Athl Train. 2014;49(6):780-5.

17. Allison KF, Keenan K, Sell TC, Abt JP, Nagai T, Deluzio J, et al. Musculoskeletal, biomechanical, and physiological gender differences in the US military. US Army Med Dep. J. 2015;Apr-Jun:22-32.

18. Lorenzo S, Halliwill JR, Sawka MN, Minson CT. Heat acclimation improves exercise performance. J Appl Physiol. (1985) 2010;109(4):1140-7.

19. Buchheit M, Voss SC, Nybo L, Mohr M, Racinais S. Physiological and performance adaptations to an in-season soccer camp in the heat: associations with heart rate and heart rate variability. Scand J Med Sci Sports. 2011;21(6):477-8.

20. Noe RS, Choudhary E, Cheng-Dobson J, Wolkin AF, Newman SB. Exertional heat-related illnesses at the Grand Canyon National Park, 2004-. 2009. Wilderness Environ Med. 2013;24(4):422-8.

21. Backer HD, Shopes E, Collins SL, Barkan H. Exertional heat illness and hyponatremia in hikers. Am J Emerg Med. 1999; 17(6):532-9.

22. Falk B. Effects of thermal stress during rest and exercise in the paediatric population. Sports Med. 1998;25(4): 221-40. 